\title{
Civilisations
}

Revue internationale d'anthropologie et de sciences

humaines

$55 \mid 2006$

Confrontations et alliances dans les Amériques autochtones

\section{Guerre et factionnalisme entre les Itzaes durant la periode coloniale}

\section{Laura Caso Barrera}

\section{(2) OpenEdition \\ 1 Journals}

Édition électronique

URL : http://journals.openedition.org/civilisations/206

DOI : 10.4000/civilisations.206

ISSN : 2032-0442

Éditeur

Institut de sociologie de l'Université Libre de Bruxelles

\section{Édition imprimée}

Date de publication : 1 octobre 2006

Pagination : 52-69

ISBN : 2-87263-10-4

ISSN : 0009-8140

\section{Référence électronique}

Laura Caso Barrera, "Guerre et factionnalisme entre les Itzaes durant la periode coloniale »,

Civilisations [En ligne], 55 | 2006, mis en ligne le 01 octobre 2009, consulté le 23 avril 2019. URL : http:// journals.openedition.org/civilisations/206 ; DOI : 10.4000/civilisations.206

(c) Tous droits réservés 


\title{
Guerre et factionnalisme entre les Itzaes durant la periode coloniale ${ }^{1}$
}

\author{
Laura CASO BARRERA
}

Résumé : Cet article analyse les relations de guerre, les migrations et le factionnalisme chez les Itzaes Maya depuis la fin de la période classique jusqu'en 1712. Les problèmes internes aux "parcialidades » (Ndlr : segments territoriaux) ont entraîné l'abandon de leurs villes et un exode migratoire. Au cours de la période coloniale, le royaume Itza établit des rapports d'alliance et de conflit avec les villes Maya peuplées de fugitifs comme Tipu. Avec les autres peuples Maya comme les Manche Chol, les Lacandons et les Petenacte, les Itzaes étaient continuellement en dispute et en guerre. Les batailles que cela impliquait visaient à contrôler les aires frontalières pour obtenir des ressources valorisées comme le sel, le cacao, la vanille, l'achiote, ainsi que pour prélever des victimes sacrificielles pour leurs dieux. Enfin, l'article souligne le rôle de la résistance et de la guerre que les Itza Maya devaient organiser contre les envahisseurs espagnols qui, en 1697, prirent le contrôle de leur ville. Les Itzaes Maya durent développer des nouvelles tactiques guerrières leur permettant d'éviter une confrontation directe avec les Espagnols. Ces stratégies ont été pauvrement analysées à ce jour.

Mots-clés : Mayas, Itzaes, guerre, résistance.

Summary: This paper analyzes the relationship of war, migration and factionalism among the Itza Maya, since the end of the classic period until 1712. The internal problems amongst the Itza "parcialidades" derived in the abandonment of their cities and their migration to other places. During the colonial period, the Itza kingdom established relationships of alignment and conflict with the Maya fugitive towns like Tipu. With other Maya people as were the Manche Chol, Lacandon and Petenacte, the Itza were in constant confrontation and war. These battles were conducted to control the Itza borderlands, to obtain valuable resources such as salt, cacao, vanilla, achiote and to take sacrificial victims for their gods. Finally this paper emphasizes the role of resistance and warfare that the Itza Maya implemented against the Spanish invaders, which in 1697 took control over their city. The Itza Maya had to develop new warfare tactics to avoid direct confrontation with the Spaniards. These strategies have been poorly analyzed until now.

Keywords: Mayas, Itzaes, war, resistance.

1. L'auteur remercie de l'appui reçu le CONACYT (Projet d'installation: «Relaciones entre mayas de Yucatan, El Petén y La Verapaz, siglos XVII-XIX »H- 38381 et le Projet de Coopération bilatérale : J200-1468), pour la réalisation et la présentation de ce travail au congrès de la Société des Américanistes de Belgique en 2002. Le texte a été traduit de l'espagnol par Viviane Merckx (CERCAL, Bruxelles). 


\section{Itzaes : guerres, migration et factionnalisme}

T es Itzaes sont un groupe maya, selon toute vraisemblance originaire de El Petén

Lqui, à la fin de la période classique, ont dû abandonner leurs villes et émigrer dans le nord du Yucatán en raison des guerres incessantes entre les grandes villes-état comme Tikal, Calakmul et Caracol qui dévastaient la région (Boot 1997, Schele et Matthews 1998). Pourtant, les Itzaes n'émigrèrent pas à des fins pacifistes. Au contraire, ils arrivèrent dans le nord de la péninsule dans le but de conquérir et de fonder un seigneuriage qui prendrait le contrôle politique, religieux et commercial de la région. Leur principal rival aurait été le seigneuriage de Cobá, suivi des villes du Puuc. Les Itzaes commencèrent par conquérir la ville de Izamal et ensuite la ville de Yaxuná, dont la chute marquerait la perte du pouvoir de Cobá et le contrôle des Itzaes sur le nord du Yucatán (Schele et Freidel 1990 : 351-353; Freidel 1992 : 102-104). On peut affirmer que, durant cette période, les Itzaes ont livré des guerres qui leur ont permis de prendre possession d'un territoire, de le contrôler et d'établir leur seigneuriage, mais également de livrer entre factions rivales des batailles qui ont provoqué la chute et l'abandon de leurs villes et la migration de retour vers leur terre d'origine. Ce type de confrontations entre clans d'un même groupe a été peu étudié jusqu'à présent et est, dans le cas des Itzaes, d'une importance capitale pour comprendre le déroulement des événements historiques les concernant.

Les migrations et les guerres des Itzaes durant la période post-classique sont restées scellées dans des documents coloniaux connus sous le nom de livres de Chilam Balam, surtout dans ce qu'on appelle les « chroniques historiques », où sont relatés leur arrivée au Yucatán, l'établissement de leurs populations ainsi que la fondation et la chute de leurs villes, et encore la migration de retour d'un faction itza à El Petén. Dans ces sources, on fait également état de la relation entre ce groupe et l'établissement du temps katunique ${ }^{2}$ et on signale que les membres de ce groupe étaient « ceux de la natte et du trône prêtés », laissant ainsi entendre qu'il s'agissait d'un groupe qui s'est emparé du pouvoir (Caso Barrera et Aliphat 2002 : 715). Dans la chronique I du livre de Chilam Balam de Chumayel, Tizimín et Maní (Barrera Vásquez et Rendón 1948 : 43-46), on relate que les Itzaes sont arrivés au Yucatán et ont découvert Chichén Itzá ${ }^{3}$ au katún $6 \mathrm{Ahau}^{4}$. Dans cette ville, ils établirent un gouvernement qui semble différer radicalement de celui qui existait dans les villes-états de la période classique. La chronique II du Chilam Balam de Chumayel décrit l'origine des Itzaes et mentionne qu'avant d'arriver à Chichén ils s'appelaient cantzuculcabes ${ }^{5}$ et qu'ils ne prirent le nom d'Itzaes qu'au moment de fonder la ville. Ce passage montre clairement que les Itzaes étaient constitués de quatre factions ou groupes et qu'au moment de fonder leur ville et d'établir leur gouvernement ils se sont rassemblés en un seul groupe. Ils ont élu un Ahau sous les ordres duquel on trouvait quatre principaux, avec lesquels il devait gouverner. On donna la dénomination de multepal ou gouvernement conjoint à ce type de gouvernement (Barrera Vásquez 1980 : 539-540; Schele et Freidel 1990 : 360-364). Les ahauoob de Chichén Itzá ont mis sur pied un art et une architecture qui légitimera

2. Ndlr : en référence au calendrier maya.

3. Chichén Itzá signifie au bord du puits naturel des Itzaes.

4. Ndlr : époque du calendrier maya.

5. Cantzuculcaboob signifie quatre factions ou parties d'un peuple. 
leur conquête et consolidera leur pouvoir. Les textes glyphiques de Chichén ne retracent pas une histoire dynastique, mais se concentrent sur les rituels de consécration pratiqués par un groupe de dirigeants. Ceci marquera une différence nette avec les monuments et inscriptions trouvés dans les villes-états durant la période classique.

Les chroniques historiques signalent que les Itzaes ont gouverné à Chichén Itzá durant deux cents ans, pour s'établir ensuite à Chakanpután, où ils arrivèrent au katún 6 Ahau (692-711). Ils y restèrent deux cents ans pour ensuite abandonner ce site au katún 8 Ahau (928-948). Selon la chronique III du Chumayel, Chakanputún fut prise par deux capitaines Itzaes appelés Kak ou Pacal et Tec Uilu (Caso Barrera et Aliphat 2002 : 717). Ceux deux personnages ont également conquis Motul et Izamal et fondé ensuite la ville de Mayapán (De la Garza, 1983 t. I : 269-270 et 305-306). Ces faits historiques mettent en évidence l'existence de deux factions Itzaes rivales, ce qui provoqua la chute et l'abandon de Chichén Itza et l'apogée de Mayapán. La faction vaincue se réfugia à El Petén, mais revint selon toute vraisemblance à Yucatán au katún 4 Ahau pour organiser des représailles contre Mayapán et la détruisit (Caso Barrera et Aliphat 2002 : 718). L'abandon de Chichén supposa la migration d'un groupe itzá vers El Petén, où ils établirent à nouveau leur seigneuriage, en fondant leur ville dans l'île de Noh Peten, dans la lagune de Chaltunha (voir carte).

L'organisation politique de Chichén Itzá fut reproduite à Tah Itza, où le Ahau Canek avait la charge d'être le " premier parmi les égaux ». Sous ses ordres, on trouvait quatre autres principaux qui représentaient les principales factions qui formaient la « nation » Itza (Caso Barrera 2002 : 217-222) (voir carte). Durant les $16^{\mathrm{e}}$ et $17^{\mathrm{e}}$ siècles, les Itzaes guerroyèrent contre des peuples voisins des environs pour étendre leurs domaines, contrôler d'importantes ressources naturelles, assurer leurs frontières et consolider leur pouvoir politique et religieux. Ils devaient également exercer un contrôle sur les populations fugitives qui fuyaient les rigueurs de la domination hispanique, en s'établissant au sud de la péninsule du Yucatán (Farriss 1984 : 199-223; Caso Barrera 2002 : 123-164). Ces peuples jouèrent un rôle très important comme frein à l'avancée hispanique en terrain itzá et comme intermédiaires commerciaux et culturels entre ce seigneuriage et les peuples de la encomienda ${ }^{6}$ (Jones 1989, Caso Barrera 2002). Les Itzaes dévastèrent également les populations mayas christianisées du Yucatán et du Tabasco, et acquirent parmi eux la réputation de guerriers féroces. Depuis 1573, les Espagnols commencèrent à réaliser des raids en vue de conquérir les Itzaes, efforts qui s'avérèrent totalement vains en raison du caractère inaccessible de la région et le caractère belliqueux de ses guerriers.

En plus des guerres qu'ils livraient avec d'autres seigneuriages mayas voisins comme les Choles del Manché, les Lacandons et les Petenactes, les Itzaes livraient également de violentes batailles à un groupe avec lequel ils étaient étroitement apparentés et qu'ils dénommaient Tulunquies ou Chinamitas ${ }^{7}$ (voir carte). Il semblerait que ce groupe était celui des Xocmoes, probablement une faction itzá dissidente établie près du fleuve Xocmo, d'où ils avaient un accès aux terres fertiles où on pouvait cultiver le cacao.

6. Ndlr: lot d'habitants indigènes placés par la Couronne espagnole sous l'autorité d'un conquérant espagnol.

7. Tulunci signifie muraille de henequen (agave). Je pense qu'il s'agit des Xocmoes, car de nombreux documents décrivent ces populations entourées de plantes de henequen pour se défendre. 


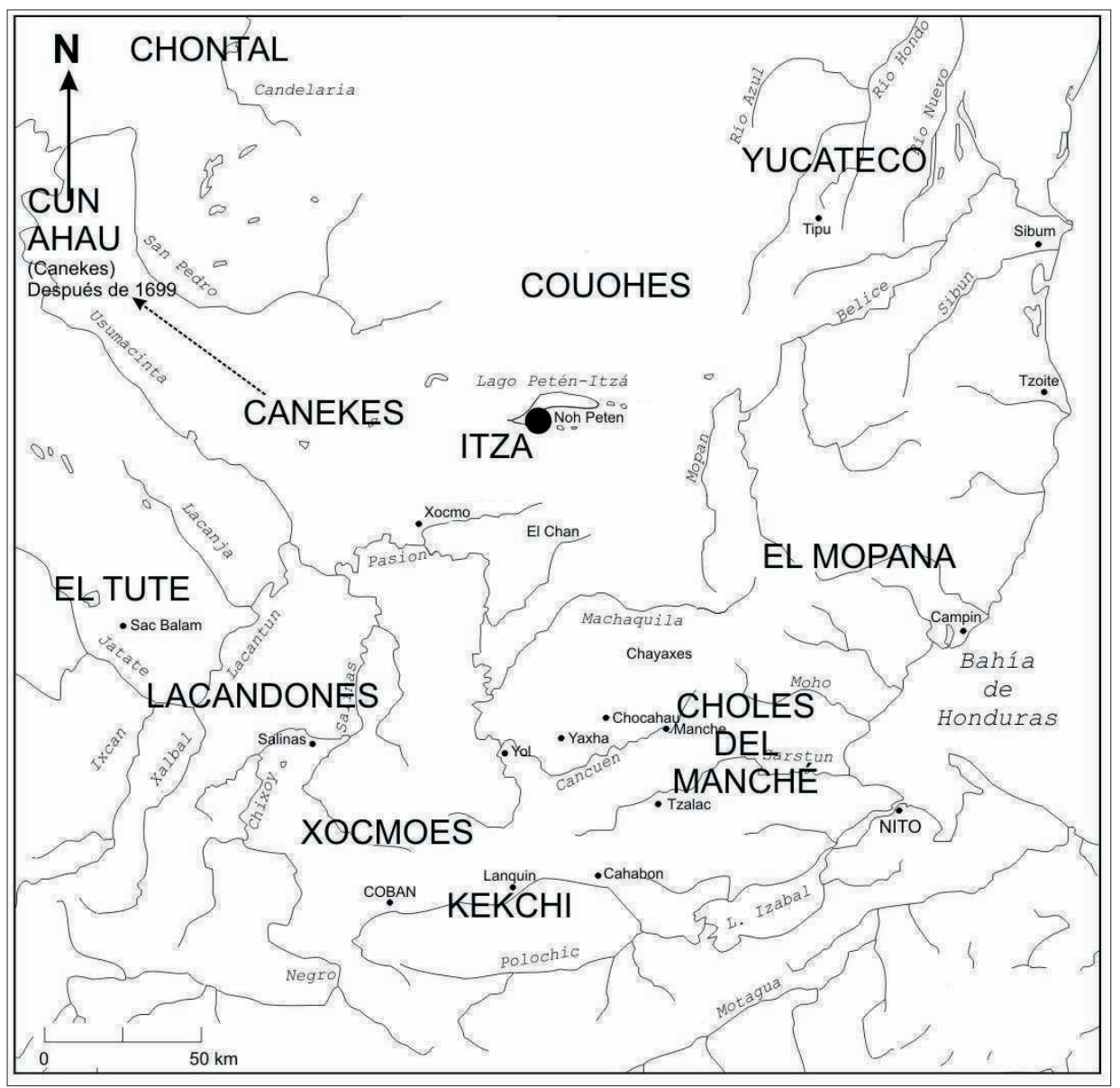

Carte des hameaux itzaes et populations mayas voisines 
Villagutierre (1933 : 380) signale ce qui suit concernant ce groupe :

Et je pense, que ces Chinamitas devaient faire partie ou être des voisins des Itzaes quand ils se trouvaient au Yucatán, qui se retirèrent avec eux ou étaient des leurs, qui avec le temps se sont divisés et sont devenus différents.

Les Itzaes remontaient le fleuve Xocmo à l'aide de nombreux grands canoës pour guerroyer contre les Xocmoes, avec lesquels ils s'affrontaient de manière féroce et ce, alors qu'il est probable que ces derniers étaient une faction d'un même groupe que le leur. Villagutierre (1933 : 380) fait référence à cela dans le passage suivant :

Parce que ces gens, non seulement se livrent d'âpres guerres les uns aux autres quand ils appartiennent à des nations distinctes, mais aussi quand ils appartiennent à la même, prenant divers caciques ou chefs, ils se lancent dans des guerres et ont pour coutume de manger leurs captifs réciproques, quand de part et d'autre ils se craignent, comme c'était le cas des Itzaes et des Chinamitas.

Frère Andrés de Avendaño y Loyola se rendit à Noh Peten en 1696 pour tenter de convaincre les Itzaes qu'ils devaient se soumettre à la domination espagnole, car il en allait ainsi dans leur prophéties. Ce moine fut le témoin d'une réunion entre Canek et les principaux des autres clans, qui refusaient que Canek tente de passer avec les Espagnols un pacte qui assujettisserait leur peuple (Caso Barrera 1996 : 44-45). Les principaux des quatre autres clans arrivèrent en canoë avec leurs officiers de guerre, qui portaient leurs insignes, leurs flèches et lances aux pointes de silex de près d'un quart de mètre de longueur, parés de plumes de multiples et voyantes couleurs. Tous avaient le visage et le corps peints en noir, portaient des tenues de guerre et étaient «emplumés » (Avendaño 1696 : 35). Avendaño en essayant de convaincre le cacique du clan Couoh que ses propres prophéties indiquaient qu'il était temps qu'ils se soumettent aux Espagnols, s'entendit répondre par le cacique « en quoi est-ce important que soit arrivé le temps pour nous d'être chrétiens, si je n'ai pas usé la fine pointe de silex de ma lance » (Avendaño 1696 : 37). La réponse du cacique Couoh synthétise la pensée Itza en ce qui concerne la soumission de son peuple : ils ne se sont jamais avoués vaincus et ont lutté avec véhémence jusqu'à la fin. Avendaño a été le témoin de la tension existant entre Canek et les principaux des autres clans, signalant que le ahau ne pouvait pas prendre de décisions sans l'avis des autres gouvernants (Caso Barrera 1996 : 45-46).

Chaque clan Itza avait ses propres capitaines de guerre, qui avaient en charge des compagnies formées par les quartiers et les factions. Il est quasi certain que ces hommes ne consacraient pas tout leur temps à la guerre, mais qu'ils s'occupaient d'agriculture et de chasse, et devenaient guerriers le cas échéant. Ils possédaient des espions et des pisteurs aidés de chiens qui servaient d'éclaireurs. Les Itzaes étaient très compétents. Ils organisaient leurs attaques en utilisant les voies fluviales, arrivant par groupes importants dans des canoës tout en bénéficiant de l'appui de groupes terrestres. Les armes qu'ils utilisaient étaient des arcs et des flèches qu'ils maniaient avec beaucoup d'adresse. Il en allait de même avec les lances, courtes ou longues, munies de pointes d'obsidienne, de silex et même de haches ou de machettes de fer, matériaux qu'ils réutilisaient de cette façon. Ils utilisaient aussi de petites palissades faites de bouts de bois effilés appelées en itzá cuman, des tranchées et des pièges avec des pointes effilées (Avendaño $1696: 28 \mathrm{v}$; Archivo General de Indias (dorénavant AGI) Patronato 237, r.5; Caso Barrera 2002 : 265). Outre tous ces moyens, les Itzaes avaient recours à des embuscades et des duperies, 
comme celles entre autres de laisser croire à l'ennemi qu'ils venaient en paix. En maya du Yucatán, ces tactiques portent le nom de hobon ch'uc oci et canupobchil cahobon (Alvarez 1997 : 578).

Les Itzaes étaient très proches de leurs divinités guerrières. La principale était Pakoc (Celui qui effraie). Il y avait aussi Ah Ox Chuncham (Celui aux trois gencives). Ils emportaient ces deux déités lors des batailles et elles se voyaient offrir du copal. Il en allait de même lorsqu'ils accomplissaient une action de bravoure. Ces déités étaient consultées avant d'entreprendre une bataille, et selon Avendaño (1696:37-37v), « elles avaient pour habitude de leur répondre, Pakoc étant celui qui parlait le plus souvent ». Ils pratiquaient des rituels durant lesquels ils dansaient et parlaient avec ces déités, et les hommes se peinturluraient «horriblement» le corps en imitant l'aspect du dieu (Villagutierre 1933 : 378). Ils vénéraient également Cun Ahau, dieu principal de la mort, à l'aspect de Vénus durant son transit dans l'inframonde. Cette planète est étroitement liée à la vision cosmique maya de la guerre et du sacrifice (Caso Barrera 2002 : 241).

\section{Tipú : entre guerre et paix}

Les Itzaes attirèrent dans leur sphère de pouvoir politique et militaire les populations composées par les fugitifs des peuples de la encomienda du nord du Yucatán. Grâce à ces peuples, ils pouvaient obtenir des produits de grande importance tels des outils de métal, haches, machettes, couteaux et sel. Mais une des fonctions principales remplies par ces peuples fut de faire barrière et de freiner l'avance espagnole en territoire itzá. Les relations qu'établirent les Itzaes avec le village de Tipú en est un bon exemple.

La population de Tipú appartenait à la province de Bacalar. Les Espagnols ont tenté durant de nombreuses années d'arriver en territoire Itza par le biais de cette population (voir carte). Depuis 1618, des religieux franciscains firent des incursions pour tenter de convertir et de soumettre les Itzaes. En 1622, le Frère franciscain Diego Delgado tenta de gagner la capitale des Itzaes en partant de Tipú. Il partit avec le cacique de ce peuple accompagné de quatre-vingts indigènes. Mais en arrivant à El Petén, ils furent tous sacrifiés par les Itzaes (Jones 1989 : 177). Il semble bien que ces derniers décidèrent que Tipú ne serait plus la porte d'entrée de leur territoire.

En 1638, Tipú et des peuples limitrophes abandonnèrent leurs positions et se rebellèrent contre la domination hispanique (Jones 1990 : 179). Tipú devint le centre de rébellion, convainquant, en les menaçant même, les autres populations de s'unir à leur cause en disant :

[...] qu'ils devaient obédience à leur roi et devaient quitter leur village, car sinon ils allaient tous mourir et périr, car un jour les Itzaes viendraient les tuer et qu'il y aurait une forte mortalité et que des ouragans menaceraient la terre (AGI, México 360, r. 29).

On remarque, pour le moins au niveau du discours, que les Itzaes se trouvaient derrière le soulèvement de Tipú et d'autres peuples. Les Itzaes semblent avoir profité de l'ouverture de la période du Katún 1 Ahau et de l'oppression exercée par les Espagnols sur ces peuples pour encourager la rébellion et éviter que ces populations ne facilitent l'invasion de leur territoire. A cette occasion les Itzaes eurent recours à l'intimidation pour que les peuples s'unissent au mouvement de résistance, menaçant ceux qui n'étaient pas d'accord. Le Katún 1 Ahau se caractérise dans les prophéties par l'arrivée de la guerre, de maladies et de famines. On prédit pour cette période la fin des gouvernants exploiteurs, qui sont associés à des animaux comme des renards, des kinkajous et des 
sarigues. On annonce la fin de l'avarice, du dépouillement et de l'exploitation, et comme l'on dit les populations qui abandonnèrent leur village, on prédit des tempêtes et des ouragans : «Pluie de tourbillons furieux sera votre lot, pluie drue, pluie fine pour le plus mauvais des katún; un jet de semences et ce sera ensemencé » (Barrera Vásquez et Rendón 1948 : 136). Les Itzaes, après avoir assuré la sécurité de leur province, voulaient contrôler la riche production de cacao des lieux. Pour les Itzaes, le cacao était un produit très important et celui-ci ne se cultivait pas bien chez eux; aussi essayèrent-ils d'y avoir accès, que ce soit par la voie diplomatique ou par l'affrontement armé.

En 1640, le gouverneur du Yucatán, Marqués de Santo Floro, décida d'envoyer des religieux franciscains pour tenter de pacifier les rebelles. On désigna pour cette tâche le Père Bartolomé de Fuensalida et Juan de Estrada, qui arrivèrent à Bacalar où ils trouvèrent les villages abandonnés, les églises brûlées et sur les chemins qui menaient à la montagne, des gros tas comme des statues à figure humaine, vêtus comme des Espagnols, alignés le long du chemin, et parmi eux des « idoles ». Selon les indigènes qui accompagnaient les religieux, « les fugitifs voulaient ainsi faire comprendre, que le chemin était coupé afin d'empêcher les Espagnols d'entrer et que leurs dieux gardaient le chemin, que ceux qui désireraient passer par là seraient enchaînés et enchantés (López de Cogolludo 1971 : 500). Il semble bien que ceci soit un rituel commun aux divers villages mayas, qui fermaient les chemins de cette manière pour empêcher les Espagnols de pénétrer sur leur territoire $^{8}$ (De Vos 1988 : 109-110).

Les rebelles s'étaient laissés pousser les cheveux; leurs corps portaient des peintures et ils utilisaient des arcs et des flèches. Le cacique du village de Holpatain, Pedro Noh, donna aux Frères un friand de poule de terre, « ce qui signifiait la guerre et la volonté de ne pas faire la paix ». Les Itzaes utilisaient également des nourritures spéciales qui symbolisaient l'animosité et le sacrifice prochain de ceux à qui cette nourriture était offerte. De plus, les indigènes accueillirent les Frères avec des danses et des beuveries. Tous étaient armés et méfiants. Ils avaient reconstitué une organisation militaire, avec des capitaines, des guerriers et des espions. Les indigènes, obéissant à leur principal qui faisait office de capitaine de guerre, attachèrent les Pères Fuensalida et Estrada et, menaçants, leur dirent : « que vienne le gouverneur, que vienne le roi, que viennent les Espagnols, nous sommes prêts à nous battre avec eux » (López de Cogolludo 1971 : 515). Les rebelles utilisaient des armements indigènes principalement des arcs, des flèches et des lances à petites pointes faites en obsidienne. On remarque ainsi qu'ils récupérèrent des techniques anciennes pour mettre au point des instruments de pierre; on sait même qu'ils réussirent à recycler des morceaux d'obsidienne de l'époque préhispanique (Simmons 1995 : 141-142). Les Tipuanos semblent avoir été fortement influencés culturellement et politiquement par les Itzaes. Ceci se reflète dans la l'organisation politique et militaire des premiers, dans leurs rituels, leurs techniques pour réaliser des armes et leur manière de faire la guerre. Ils récupérèrent ainsi des rituels et des symboles itzaes liés à la guerre, tel que l'utilisation de carapaces de grands escargots comme trompettes, dont ils jouaient pendant les batailles, des repas spéciaux et une bataille corps à corps qui avait lieu avant la guerre et qui était désignée sous le nom de pech ni, ce qui signifie " aplatir le nez » (Caso Barrera 2002 : 180-181).

8. Voir le cas des Lacandons in Jan de Vos, 1988 : 109-110. 
Il semblerait bien que parmi les insurgés on trouve des Itzaes qui soutenaient les gens de Tipú dans leur lutte contre les Espagnols. S'il est vrai que les Tipuanos et les Itzaes établirent des liens politiques et commerciaux solides, ils n'en connurent pas moins entre eux des moments de conflits, générés apparemment par le pouvoir et l'autonomie obtenue par Tipú. Il est possible que les Itzaes aient essayé de maintenir un contrôle plus important sur ce peuple, lequel résista selon toute vraisemblance en essayant de conserver l'indépendance ainsi que le pouvoir politique et économique dont il jouissait. López de Cogolludo signale que de 1655 à 1656 ces deux peuples étaient en guerre, guerre qui fut gagnée par les Itzaes.

Dans la province de Bacalar, les Itzaes ont réussi à utiliser les populations fugitives comme tampon. Ces populations continuèrent cependant à servir d'intermédiaires entre les Itzaes et la domination coloniale. Leur fonction commerciale était vitale; grâce à elle, les Itzaes obtenaient des outils de métal et des informations sur les événements du Yucatán et surtout sur les plans et les mouvements des Espagnols. Grâce aux contacts qu'ils maintenaient avec ces groupes, les Itzaes ont su comment faire face aux Espagnols et à leur armement. L'arrivée du Katún 8 Ahau en 1697 annonçait un changement politique pour les Itzaes. La conquête de ceux-ci par les Espagnols affecta les peuples fugitifs avec lesquels ils maintenaient des relations et des alliances, comme ce fut le cas de Tipù. Après la conquête des Itzaes, les Tipù fuirent vers la forêt pour s'unir à la résistance contre l'invasion hispanique.

\section{Guerres avec d'autres seigneuriages mayas}

Les Itzaes livrèrent d'incessantes guerres contre des groupes voisins comme les Choles du Manché, les Lacandons, les Petenactes et les Xocmoes (voir carte). Ces guerres avaient divers objectifs. Ils attaquaient ces groupes, surtout les Choles, quand ceux-ci acceptaient la christianisation des Pères dominicains, ce qu'ils voyaient comme une menace et un prélude à une avancée hispanique sur leur territoire. Les Itzaes attaquèrent par surprise le village de Chol de San Miguel en 1630, tuant leurs principaux et faisant plus de trois cents prisonniers. Le gouverneur principal Martín Cuc fut sacrifié et «mangé ». En conséquence de l'attaque itzá, onze villages Choles se rebellèrent et refusèrent l'obéissance aux Espagnols, abandonnant leurs populations (Tovilla 1960 : 265).

Ils ont mené des guerres continues ceux du Manché avec ceux de Ah Itza, mais ils ont toujours été perdants parce qu'ils sont peu nombreux et ceux de Ah Itza nombreux, et ainsi chaque année au cours du yazquin, qui est l'été, certains d'entre eux se font emmener comme prisonniers, comme cela s'est passé l'an dernier l'année 1630 au cours de laquelle furent capturées cent personnes, et ainsi ils en prennent l'habitude, et comme ils sont chrétiens ces Manché, ils les pourchassent surtout parce qu'ils confinent à leurs terres, et qu'ils veulent les empêcher de renseigner les espagnols sur le chemin à suivre parce qu'ils craignent qu'ils ne les punissent pour leur effronterie et les méchancetés qu'ils ont commises, notamment lorsqu'au cours de l'année 1624, ils tuèrent le capitaine Mirones et les vingt soldats qui l'accompagnaient, lequel était arrivé par Yucatán, qui est la terre des espagnols la plus proche de la leur (Tovilla 1960 : 185).

Parce que bien que les Indiens de la nation chol sont réductibles, car dociles, il y a perdue dans les collines une nation différente appelée ah itza, de nature indomptable et qui va en chasse de chair humaine pour s'enduire d'elle, alors que ses membres refusent résolument le saint baptême et veulent en interdire l'accès aux choles voisins (AGI, Guatemala 179). 
Les Itzaes livraient également des batailles pour le contrôle des ressources naturelles et à des fins politiques et religieuses, comme celle d'obtenir des victimes pour le sacrifice. Avec la conquête espagnole et la chute du seigneuriage chontal de Acalán, les Itzaes sont devenus le centre qui donna l'impulsion au commerce du cacao et des produits associés à sa consommation, comme le rocouyer et la vanille, commerce sur lequel ils exercèrent leur domination. De plus, ils contrôlaient le commerce avec les peuples fugitifs, grâce auxquels ils obtenaient leurs outils de métal et le sel. Comme on l'a vu pour Tipú, les Itzaes étaient surtout intéressés par l'accès aux riches plantations de cacao (Caso Barrera $2002: 230)$.

Tandis que les peuples fugitifs étaient subordonnés politiquement et commercialement aux Itzaes, d'autres peuples mayas luttaient pour conserver leur autonomie, comme les Choles, les Lacandons et les Petenactes. Les Itzaes obligeaient les Choles et les Lacandons à commercer avec eux et à les aider à obtenir du cacao, du rocouyer et de la vanille. En cas de refus, ces populations étaient victimes de lourdes représailles (AGI, Guatemala 152).

Prenons un cas précis, celui des Lacandons. Ceux-ci étaient des ennemis naturels des Itzaes qu'ils affrontaient fréquemment pour le contrôle des salines de Los Nueve Cerros (Bolon Uitz), source unique de ce minéral dans le nord du Guatemala (León Pinelo 1960 : 263). Quand les Lacandons furent obligés de se rendre en 1586 vers une région plus accessible et s'établirent à Sac Balam, ils commencèrent à perdre des membres de leur population et à s'affaiblir, ne pouvant plus résister aux assauts des Itzaes. Ceux-ci les obligèrent à commercer avec eux pour le cacao, le rocouyer et le noir de fumée qui leur servait à écrire. Finalement, les Itzaes prirent le contrôle des salines, et ce minéral devint un des plus importants articles d'échange.

Les affrontements armés permettaient non seulement aux Itzaes d'avoir le contrôle politique et économique de la région et d'assurer la sauvegarde de leur territoire, mais aussi de capturer des personnes dont ils se servaient comme esclaves et comme victimes pour les sacrifices. Au mois de Yaxhkin (mois de sécheresse), ils attaquèrent les Choles du Manché, les Lacandons, auxquels ils avaient l'habitude de s'attaquer la nuit, ainsi que les Petenactes et même des peuples chrétiens (Tovilla 1960 : 185; AGI Guatemala 152, f. $898 \mathrm{~V})$. Nous ne sommes pas certains que les guerres entreprises par les Itzaes contre d'autres groupes mayas eurent toutes lieu durant l'époque des sécheresses; mais comme le signale Hassig, il est probable que les conflits correspondaient aux périodes de récoltes et de fin de saison de pluies (Hassig 2000 : 166). Nous savons que, depuis le $16^{\mathrm{e}}$ siècle, les Itzaes possédaient une élite spécialisée dans les affaires de guerre; nous ne pouvons cependant pas affirmer qu'ils possédaient une « armée » à temps plein, mais plutôt qu'ils recrutaient parmi le commun des mortels quand le besoin de guerre se faisait sentir.

Les Itzaes avaient pour habitude d'arracher le cœur des victimes sacrifiées, de les décapiter, de piquer leurs têtes sur des pieux et d'arracher les membres de leur corps. De nombreuses sources rapportent qu'ils mangeaient les corps rôtis ou bouillis des personnes sacrifiées (Caso Barrera 2002 : 244-245; AGI Guatemala 151 Bis, f. 545). Les documents historiques relatent souvent que les Itzaes recherchaient des victimes pour les sacrifier et les manger. Ceci pourrait apparaître comme une exagération ou un préjugé des chroniqueurs espagnols. Pourtant une analyse sérieuse des pratiques de ce groupe nous montre que le cannibalisme rituel était d'une haute importance chez eux. Equarrir les corps des personnes sacrifiées, pour ensuite les bouillir ou les rôtir en vue de les consommer lors de banquets rituels, constitua une pratique commune dans toute l'Amérique centrale et eut son apogée durant la période post-classique (Pijoan et Mansilla 1997 : 197). 
Au nombre des populations les plus harcelées par les Itzaes on trouve les Petenactes, qui étaient installés dans le Partido de los Ríos à Tabasco. Il s'agissait très probablement de Lacandons qui émigrèrent et devinrent des ennemis de leur propre groupe ainsi que des Itzaes. Les Indiens du village de Yucum, peut-être des Petenactes, affirmèrent être peu nombreux en raison de la « persécution des Indiens de Ah Itzá qui mangèrent les habitants de leur village » (AGI Guatemala 344, f. 85v). En 1694 un groupe itzá, sous les ordres du beau-frère de Canek, arriva en canoë à Tabasco au village de Canizan, village d'Indiens chrétiens, pour trouver des personnes pour les sacrifier. Les gens de ce village tuèrent le beau-frère de Canek et les Itzaes capturèrent alors le cacique Andrés Dzib, dont la fin dut être terrible (AGI Guatemala 151, f. 828). Ainsi, il nous est permis d'affirmer que la guerre avait également d'importantes connotations sociales et religieuses, et servait notamment à acquérir des victimes en vue de les sacrifier aux dieux Itzaes.

\section{Guerre et résistance contre les Espagnols}

Les Itzaes exercèrent une opposition tenace aux Espagnols, menant à partir de leur seigneuriage de El Petén des actions contre leur domination coloniale. Ils soutinrent même ouvertement la rébellion, comme nous l'avons vu dans le cas des populations fugitives et à Tipú. Ils détruisirent des populations indigènes christianisées pour éviter que les Espagnols n'entrent sur leur territoire. On peut même affirmer qu'ils ont utilisé en leur faveur les rigueurs de l'exploitation coloniale, en encourageant les Mayas du nord du Yucatán à abandonner leurs villages, et en les utilisant comme leurs intermédiaires commerciaux et comme frein à l'avancée espagnole (Caso Barrera, 2002 : 170). Finalement, les Itzaes devinrent une menace qui ne pouvait être tolérée plus longtemps par la Couronne et les autorités coloniales, d'autant plus que ces dernières espéraient conquérir une terre pleine de richesses et de minéraux précieux en vue de redresser leur trésor public fort appauvri. En 1695, deux expéditions armées partirent du Guatemala et du Yucatán pour se retrouver à El Petén afin d'aller conquérir les Itzaes. L'expédition qui quitta le Guatemala et était commandée par le capitaine Juan Díaz de Velasco tomba sur un groupe d'espions Itzaes qui suivaient de près les faits et gestes des Espagnols. Les Itzaes se battirent contre les envahisseurs avec leurs arcs et leurs flèches et au corps à corps. Les Itzaes eurent le dessus grâce à leur adresse et à leur courage. Ce type de combat, à savoir le combat corps à corps, se désigne en maya du Yucatán par le mot p'izba, qui signifie littéralement se mesurer. La description de cet affrontement est le suivant :

Et encerclant les nôtres, ils commencèrent [les Itzaes] à tirer des pluies de flèches; si les nôtres ne portaient pas de cottes, ils auraient tous péris. Ils tirèrent avec leurs fusils; mais un Indien blessé quatre fois par balle refusait de se rendre. Les nôtres mirent pied à terre et se lancèrent eux, quatre des nôtres n'étant pas suffisants pour un Indien. Un homme était traversé par une lance avec laquelle il transperça un soldat et lui donna un coup de machette sur la tête. Bien que la blessure de l'Indien ne soit pas grave, ils donnèrent à ce dernier le coup de grâce et le tuèrent. Un autre soldat s'empara d'un Indien et le soldat lui enfonça la machette dans le corps et pourtant l'Indien fit tomber le soldat à terre et manqua de tuer le soldat.

La bataille dura une heure et Machuca affirma que les Indiens étaient tellement valeureux que, quatre hommes, mêmes bons, ne suffisaient pas contre un de ces Indiens Ahitzaes (Ximénez 1973 T.V. : 358).

Ces premières rencontres donnèrent une indication aux Itzaes sur les armes et les manières de combattre des Espagnols. Aussi évitèrent-ils ce genre de confrontation directe 
et eurent recours aux pièges et aux embuscades. En 1696, une compagnie placée sous les ordres du capitaine Alonso García de Paredes, venant du Yucatán et faisant route vers La Laguna de El Petén, se trouva aussi face aux Itzaes. Cette fois-là, les Itzaes déclarèrent « être en paix », et permirent aux Espagnols de s'approcher du bord de la lagune. Là, des guerriers se rassemblèrent autour des soldats en les séparant et en les faisant monter sur leurs canoës. Les Espagnols déclarèrent que le but recherché par les Itzaes était de les faire monter sur les canoës pour les amener au milieu de la lagune et leur envoyer des flèches. Les Itzaes tentèrent alors de désarmer les soldats et réussirent à en égorger un, tandis que des mangroves sortirent une centaine d'Indiens munis de flèches, cachés jusque-là sur leurs canoës, qui attaquèrent les hommes de García de Paredes (AGI Patronato 237, r.5).

Après les affrontements violents de 1695 et 1696 entre les Itzaes et les compagnies militaires du Yucatán et du Guatemala, il fut décidé de mener une opération militaire pour « pacifier» complètement la nation Itza. Le gouverneur Martín de Ursúa partit de Yucatán. Son but était la réduction de ce groupe. Il établit un campement non loin de la lagune de El Petén, où il ordonna la construction d'une goélette et de deux pirogues afin de prendre la capitale des Itzaes. On vit arriver diverses ambassades Itzaes au campement annonçant qu'elles voulaient la paix avec les Espagnols, ce qui semble avoir été une manière de se rapprocher des forces et des armements espagnols. Ils envoyèrent aussi des femmes Itzaes pour distraire les soldats et pouvoir les attaquer par surprise. Mais les Espagnols ne tombèrent pas dans le piège (AGI Guatemala 151 Bis, ff. 519-524). Envoyer des femmes était apparemment un piège couramment utilisé par les Itzaes. Les femmes arrivaient en même temps que des groupes de guerriers qui s'approchaient du campement en bravant les Espagnols tout en jouant de leurs tunkules ${ }^{9}$ et de flûtes et en poussant des cris pour essayer de déclencher le combat, ce qui en maya se dit pay bateel ou appeler à la guerre (Villagutierre $1933: 360$ ).

Quand finalement Martín de Ursúa s'embarqua avec ses troupes pour Noh Peten, la capitale itzá, il se retrouva face à des flottes de canoës qui lui barraient le passage et lui envoyaient une pluie de flèches. Toutefois, les Itzaes ne se heurtèrent pas de front à l'envahisseur, les Espagnols découvrant une ville déserte, car la population entière s'était réfugiée dans la forêt d'où ils organisaient la résistance (Caso Barrera 2002 : 282). Les Itzaes optèrent pour une « guerre de guérillas », attaquant l'ennemi par surprise et brûlant leurs greniers et leurs champs de maïs, compromettant de ce fait leur approvisionnement. Néanmoins, ces actions finirent par les affecter eux-mêmes, surtout lorsqu'ils durent affronter les maladies apportées par les Espagnols.

Une fois que Martín de Ursúa s'empara de la capitale des Itzaes, Noh Peten, le seigneuriage Itza fut divisé en quatre quartiers principaux composés respectivement des Canekes, des Tutes, des Couhes et des Panaes, qui commencèrent à se disputer entre eux pour le pouvoir, ce qui les empêcha de faire front commun face aux Espagnols (voir carte). Les quartiers qui opposèrent la résistance la plus hardie contre les envahisseurs furent ceux des Tutes et des Panaes, qui pratiquaient des embuscades contre les escouades de soldats qui s'attaquaient à leur champs de maïs (Caso Barrera 2002 : 308). Un des caciques parmi les plus respectés et craints appelé Pana fut fait prisonnier durant une de ces escarmouches et envoyé au bagne où il tomba gravement malade. Alors qu'un des

9. Tunkules sont des tambours horizontaux. 
moines le poussait à se baptiser, la seule chose qu'il fit fut d' " enlever ses boucles d'oreille qu'ils utilisaient comme signe ostentatoire de leur autorité de principal supérieur » pour leur faire comprendre leur défaite et mourut ensuite (American Philosophical SocietyReina-Jiménez Collection).

En 1702, les Itzaes continuaient à lutter et à tenter de chasser les envahisseurs de leur capitale et de leurs terres. Certains clans s'unirent pour tenter d'en finir avec les Espagnols. Ils construisirent des tranchées et des fortifications avec des pieux et des pierres et pensaient à nouveau pouvoir tromper les Espagnols en leur envoyant des femmes qui leur diraient « nous venons en paix et dans de bonnes intentions », alors que les hommes attendraient cachés que les Espagnols embrassent les femmes pour les tuer.

Les Itzaes utilisèrent toutes les ressources à leur portée pour se libérer des Espagnols, comme le signale le bachelier Francisco de San Miguel en 1702 :

[ ] les Indiens voulaient en finir avec ce récif avec empressement, rébellion et ruse; ce n'est qu'au hasard qu'ils doivent d'avoir réussi leur tentative empressée et rusée et c'est chance pour eux qu'ils aient pu traverser la lagune à la nage et de nuit pour atteindre ce récif (AGI Escribanía 339B).

Les Itzaes ne se sont jamais avoués vaincus, même lorsque les Espagnols parvinrent à capturer certains de leurs principaux et à réduire certains de leurs peuples. Très vite ils se rebellèrent, espérant pouvoir prendre le contrôle de la ville occupée par les Espagnols. Le 7 septembre 1704, huit des onze peuples que les Espagnols avaient pu réduire se rebellèrent et tuèrent six soldats espagnols pour fuir à nouveau dans les montagnes. Les causes de cette rébellion semblent être diverses. Tout d'abord, le capitaine Joseph Aguilar, gouverneur du bagne, déclara avoir demandé à tous les peuples leur coopération pour construire une église plus grande. Pour ce faire il leur avait demandé de couper du bois et du guano et de retourner au travail puisque, étant si nombreux, cela ne pouvait ni les fatiguer, ni leur peser. Il leur dit qu'ils pouvaient terminer de labourer, mais, selon lui, les Itzaes répondirent qu'ils préféraient commencer directement les travaux, se montrant bien disposés et très désireux de répondre à la sollicitation du gouverneur. Aussi, ce dernier les récompensa-t-il avec du sel et de la verroterie. Il se peut que la manière de leur demander de réaliser ces travaux ne fût pas aussi aimable et complaisante que ne le dit le capitaine, et que les Itzaes décidèrent, face à ses exigences, de se rebeller. Il est également très probable que les Indiens profitèrent de l'occasion que leur fournissaient les travaux au bagne pour y déclencher une rébellion et en terminer avec les gardiens du bagne. Selon un brigadier, les Indiens avaient programmé leur soulèvement trois mois auparavant dans l'idée de « tuer tous les Espagnols afin de rester maîtres de leurs terres » (AGI Escribanía $339 \mathrm{~B}, \mathrm{f} .824$ ). Le moment semblait fort propice, le brigadier étant occupé à coordonner les travaux de construction de l'église et six soldats ayant été envoyés dans le Yucatán, six au Guatemala et d'autres à surveiller les labours et le séjour du roi, alors que d'autres avaient pris la grande route pour Campeche afin de trouver un nouveau vicaire. Un soldat affirma que l'ordre de l'évêque de Yucatán de recenser les nouvelles populations fut déterminant. Ce recensement fut effectué par le Père Diego de Rivas, le brigadier et le vicaire Marcos de Vargas. Les indigènes crurent qu'on les comptait pour leur imposer un tribut, ce qui ne leur plut pas du tout. En réalité, ce sont toutes ces causes conjuguées qui ont entraîné la rébellion.

Les faits se produisirent quand le brigadier manda une galiote avec dix hommes et le pilote pour ramener du bois et des personnes au village de San Martín en vue de 
commencer les travaux de l'église. L'embarcation s'arrêta dans le village de San Antonio pour y dire que ses habitants devaient envoyer des gens pour travailler le lendemain. On reçut les visiteurs avec beaucoup de joie, et les Indiens emmenèrent cinq soldats dans diverses maisons en leur offrant des bananes, des patates et d'autres choses à manger. Les soldats demandaient toujours ces friandises lorsqu'ils allaient dans les villages des Itzaes; aussi n'étaient-ils pas étonnés qu'on les invite dans les maisons. Nous remarquons à nouveau les tactiques Itzaes, à savoir tromper l'ennemi en le mettant dans une situation vulnérable. Les soldats furent surpris alors qu'ils mangeaient et tués à coups de machette. Pendant ce temps, on apportait aussi de la nourriture dans une maison voisine pour les six hommes restés à surveiller le bateau. Ces derniers ne se laissèrent pas abuser et, alors qu'ils se mirent à rappeler leurs compagnons, ils furent attaqués. Ils réussirent toutefois à se défendre et à remonter sur la galiote. Entre temps, ayant terminé leur garde, trois autres Espagnols partaient en canoë acheter du maïs au village de San Martín. Alors qu'ils avaient débarqués, ils furent attaqués et l'un d'eux tué. Les deux autres se défendirent en tuant un des agresseurs et en en blessant d'autres, et parvinrent à atteindre le canoë. Les gens de la galiote ainsi que ces derniers rentrèrent au bagne pour informer le capitaine des événements. Celui-ci envoya immédiatement vingt hommes pour punir les rebelles, mais ils ne trouvèrent que des soldats morts. En effet les habitants de huit villages s'étaient réfugiés dans les collines (AGI Escribanía 339 B, f. 824 v.). Le brigadier envoya immédiatement dix hommes garder les labours de peur que les Indiens ne tentent d'y mettre le feu, ce qui arriva; mais les Indiens furent repoussés par deux décharges d'émeris.

Douze jours plus tard, le capitaine Aguilar réussi à capturer le cacique Pedro Dzin du village de San Jerónimo; il était un des leaders de la révolte. Ce cacique était « fort aimé » des Espagnols et était considéré comme l'un des plus coopératifs. Il déclara que lui et le cacique Citam Couoh, du village de San Joseph, avaient planifié le soulèvement, et que parmi les autres personnes impliquées se trouvaient Culut Couoh, cacique de San Martín et le cacique Kixan, du village de San Antonio (AGI Escribanía 339 B, f. 823 v). Quand on demanda à Pedro Dzin les causes de leur rébellion, il répondit ce qui suit :

[ ] sans pouvoir ni s'excuser de cette altercation, ni en donner la raison, si ce n'est que c'est le diable qui les avait trompé, c'était toujours sa faute. C'est qu'une nouvelle s'était propagée parmi eux que l'on voulait les contraindre à quitter leurs milieux naturels et les envoyer peupler le royaume du Guatemala ainsi que la Province du Yucatán.

Ils savaient également que dans ces deux provinces on paie un tribut aux Espagnols, ce qui est une corvée pour eux. Ils craignaient, vu leur peu de foi et leur faible capacité de raisonnement, que ces désordres ne leur apporte le Mal. Pourtant il est de notoriété publique que nous les traitons bien [ ] (AGI Escribanía 339 B, f. 825).

Les six Indiens capturés par le capitaine Aguilar et qui avaient avoué (qui s'étaient confessés) furent condamnés à mort pour que ce châtiment serve de " grand exemple » face aux menaces faites constamment par les principaux «à savoir qu'il ne fallait pas qu'il reste un seul Espagnol vivant et qu'il fallait tuer les messagers qui sortaient de ce bagne ou y entraient» (AGI Escribanía 339 B, f. 825). Ils furent tous les six fusillés et leur exécution servit d'exemple pour les Indiens qui restèrent dans les villages de San Joseph, San Andrés et San Miguel. Il ne restait alors que trois cents familles dans les trois villages. Ensuite, vingt cinq autres familles fugitives revinrent. Le brigadier ne fut pas en mesure de partir à la recherche des autres fuyards, car il n'avait pas suffisamment d'hommes sous 
la main. Aussi, demanda-t-il à l'Audiencia ${ }^{10}$ d'envoyer cinquante soldats de plus, de la poudre, des balles, vingt-cinq mules et la solde due à l'infanterie afin de lui permettre de se procurer ce dont ses hommes avaient besoin et de rembourser à leurs créanciers leurs dépenses en matière de vêtements et autres. Après le soulèvement, le capitaine Aguilar appliqua une politique plus sévère envers les principaux indigènes. Aussi demanda-t-il ceci :

Il nous faut également deux cents chaînes d'une aune de long avec les attaches pour les pieds, qui nous serviront pour les pères de familles et les chefs de famille, surtout ceux ayant un bec de lièvre, car ils sont très attachés à leurs vices, à savoir se tuer entre eux quand ils sont ivres, avoir chacun trois ou quatre femmes, ce qui est inadmissible pour nous. Le pire est qu'ils sont frères entre eux, qu'ils n'aiment pas être séparés ou se disputent, et selon leurs paroles respectives, ils restent ou ils partent, d'où en les gardant prisonniers nous nous ferons maîtres de leurs personnes et de leurs volontés et nous les endoctrinerons à l'aise et en toute sécurité. Ainsi prisonniers, ils feront leurs labours et leurs maisons parce qu'ils sont lâches et ne veulent même pas travailler pour eux-mêmes. Et si jusqu'ici, bon Dieu, ils ont fait peu de cas de ma prévenance et de mon affection pour eux, connues de tous et dont le révérendissime Frère Diego de Rivas, qui je crois est dans cette ville, peut témoigner devant Votre Seigneurie, alors que je leur témoignais autant d'amour qu'un père. Et comme, malgré tout, ils n'en ont pas voulu, même dans leurs propres intérêts, il faudra qu'on les amène à la véritable connaissance (AGI Escribanía 339 B, f. 826-826v).

Ces principaux ou chefs de clans ont dû se mettre en valeur par des actions d'éclat puisque la partie inférieure de leur lèvre était fendue pour utiliser des ornements. Beaucoup d'entre eux étaient des parents proches, ce qui ne les empêchait nullement de s'affronter. Ceci est le reflet de la situation politique chez les Itzaes. Les moyens utilisés pour contrôler ces individus, les obliger à rester dans leurs villages et à travailler, semblent fort extrêmes et certainement mal acceptés de leur part.

En 1712, trois peuples Itzaes, qui avaient réussi à conserver leur autonomie, commerçaient avec quelques Anglais, avec lesquels ils échangeaient des armes pour des Indiens captifs que les Anglais emmenaient comme esclaves en Jamaïque. Selon les dires des Anglais capturés par le capitaine Joseph Galeano, les Itzaes étaient désireux d'envahir le domaine de El Petén et d'en expulser les Espagnols (AGI Guatemala 186). Comme nous l'avons dit précédemment, les Itzaes livrèrent constamment une lutte féroce contre les envahisseurs hispaniques. Toutefois, leurs luttes internes les empêchèrent de mener la guerre en front commun, et ils furent décimés par les maladies apportées par les Espagnols. Comme à d'autres moments de leur histoire, les Itzaes succombèrent à leur propre factionnalisme, comme le signale le livre de Chilam Balam :

[ ] alors commença l'exode de Itza, Sorcier de l'eau, et vint le conflit avec violence, le conflit sans miséricorde (Barrera Vásquez et Rendón 1948 : 223).

\section{Conclusions}

Ce travail montre l'étroite relation existant entre les Itzaes, la guerre et la migration depuis la fin de la période classique. Il semble que l'aire occupée par les Itzaes durant cette période était située entre les territoires politiques de villes-états, comme Calakmul et Tikal. Celles-ci étaient en guerre perpétuelle entre elles et avec d'autres villes importantes

10. Ndlr : division administrative coloniale. 
comme Caracol. Il est probable que la tension croissante dans la zone en raison de conflits constants a poussé les Itzaes à migrer vers le nord de la péninsule du Yucatán.

Les Itzaes arrivèrent au Yucatán pour prendre possession d'un territoire et pour construire un seigneuriage qui prendrait le contrôle politique, économique et social de cette région. Pour atteindre leurs objectifs, les Itzaes furent obligés de se confronter au seigneuriage de Cobá et aux villes du Puuc, sur lesquels ils eurent le dessus. La consolidation de leur seigneuriage se concrétisa par la fondation de la ville de Chichén Itzá. Ils y établirent une forme particulière d'organisation politique, que l'on a appelée multepal ou gouvernement conjoint, qui représente une confédération de factions présidées par un ahau "primus inter pares ». Ce personnage maintenait un équilibre de forces dans lequel on observait un protocole rigoureux en matière de titres et de rangs. Les livres de Chilam Balam relatent les migrations des Itzaes, la fondation et la chute de Chichén Itzá, de même que celle de Mayapán, mais ont accordé peu d'importance au factionnalisme interne de ce groupe, cause de ces événements. Cette étude a mis l'accent sur l'équilibre délicat qui devait exister dans l'organisation politique itzá. La rupture de cet équilibre fut à l'origine des luttes intestines qui amenèrent la chute de leurs villes et la migration en dehors de leur territoire. Cette lutte entre factions est fondamentale pour comprendre les événements postérieurs à la conquête hispanique de 1697.

Les Itzaes exercèrent une influence sociale, politique et militaire sur les populations mayas fugitives comme Tipú, laquelle en 1638 se rebella contre la domination espagnole en grande partie sous la pression du seigneuriage itzá. Les Itzaes voulaient contrôler ce peuple, d'une part, pour empêcher l'avancée espagnole sur leur territoire et, d'autre part, pour avoir accès aux ressources aussi importantes que le cacao, le sel et les outils en métal. On remarqua le contrôle qu'exerçaient les Itzaes sur le temps katunique, l'utilisant pour encourager la rébellion des peuples de fugitifs, comme celui de Tipú en 1638, avec l'établissement du Katún 1 Ahau. Non contents de manipuler le calendrier, les Itzaes ont contribué à ce que leur propre organisation politique, militaire et religieuse soit reprise à son compte par la population de Tipú. Il fut toutefois démontré qu'en dépit de la proximité de ces deux populations, celles-ci eurent des conflits entre elles. Il est probable, en effet, que Tipú veuille plus d'autonomie. Ainsi, entre 1655 et 1656, les deux populations s'affrontèrent; mais les Itzaes finirent par avoir le dessus. Enfin, avec l'arrivée des Espagnols, les gens de Tipú abandonnèrent leurs villages et se réfugièrent dans la forêt, s'unissant à la résistance itzá contre les envahisseurs.

Les Itzaes contrôlaient non seulement les populations fugitives, mais ils menaient des guerres constantes contre des groupes voisins, principalement les Choles del Manché, les Lacandons et les Petenactes, qu'ils attaquaient pour assurer les limites de leur territoire et empêcher l'avance espagnole. Ils attaquaient surtout ces populations à l'arrivée dans celles-ci des religieux catholiques qui voulaient les christianiser. Ils entreprenaient aussi des guerres qui leur permettaient de faire du commerce avec d'autres peuples, principalement le commerce du cacao, du rocouyer et de la vanille, produits d'une importance capitale. Le contrôle des salines de Nueve Cerros provoqua des affrontements constants avec les Lacandons, jusqu'à ce que les Itzaes réussissent à s'approprier de cette ressource. Enfin, l'étroite relation existant entre la guerre et le sacrifice devient évidente. Comme d'autres peuples de l'Amérique centrale, les Itzaes devaient apaiser leurs dieux par le biais d'offrandes et de sacrifices humains. Pour le $a h a u$, chef des principaux, et pour ces derniers, leurs victoires militaires prouvaient leur capacité militaire et politique. Ils la devaient à leurs dieux qu'il fallait récompenser à travers des sacrifices. 
Tah Itzá devint le centre politique. Celui-ci encouragea la résistance et la rébellion contre la domination espagnole, se transformant ainsi en une véritable menace, à laquelle la Couronne mit fin par le biais de la conquête. En 1695 des expéditions armées quittèrent le Yucatán et le Guatemala pour se regrouper à El Petén en vue de pacifier le seigneuriage itzá. Très rapidement les Itzaes se rendirent compte qu'ils ne pouvaient pas utiliser leurs tactiques et leurs formes de guerres contre les Espagnols, car les armes à feu empêchaient les attaques directes. Aussi adoptèrent-ils de nouvelles stratégies qui ont été peu étudiées jusqu'à présent, comme l'utilisation de la ruse, de pièges et de diversion afin de gagner du temps et d'éviter l'affrontement direct. Les Espagnols prirent la capitale itzá Noh Peten en 1697. Ils la trouvèrent vide car tous les habitants avaient fui dans la forêt. La conquête espagnole coïncida avec le Katún 8 Ahau, qui marquait un changement politique interne; elle provoqua le démantèlement du seigneuriage itzá, en quatre factions principales. Dès ce moment, ces dernières commencèrent à lutter entre elles pour nommer un nouveau ahau et reconstruire le seigneuriage. Le factionnalisme itzá ne leur permit pas de faire front commun contre les Espagnols. Toutefois aucune faction ne s'unit avec les conquistadors et chacune d'entre elles se battit férocement. Ils eurent finalement recours à une " guerre de guérillas » constante et sans trêve contre les envahisseurs, qui ne réussirent jamais à soumettre la valeureuse « nation itzá ».

\section{Archives consultées}

Archivo General de Indias (AGI).

American Philosophical Society-Reina-Jiménez Collection.

\section{Références bibliographiques}

Alvarez, Cristina, 1980-1997. Diccionario etnolingístico del idioma maya yucateco. 3 vol. México : UNAM.

Avendaño Y Loyola, Andrés de, 1696. Relazion de las dos entradas que hize a la conversion de los gentiles Ytzaex y Cehaches. Ms. 1040. Edward E. Ayer Collection, Newberry Library. Chicago.

Barrera VÁsquez, Alfredo (dir.), 1980. Diccionario maya Cordemex. Mérida : Cordemex.

Barrera Vásquez, Alfredo et Silvia Rendón, 1948. El libro de los libros de Chilam Balam. México : Fondo de Cultura Económica.

Воот, Erik

1997. « No Place Like Home: Maya Exodus. Itsá Maya Migrations Between ca. A.D. 650 and A.D. 1450 » in Henri J. M. Claessen et Han F. Vermulen (eds). Veertig jaren onderweg, pp. 165-187. Leiden : DSWO Press.

Caso BARRERA, Laura

1996. « Discurso evangélico y conversión. Fray Andrés de Avendaño y la conquista del Itzá (1695-1697) », Dimensión Antropológica, vol. 7, pp. 32-54.

2002. Caminos en la selva. Comercio Migración y resistencia. Mayas yucatecos e Itzaes siglos XVII$X I X$. México : El Colegio de México-FCE.

Caso BarRera, Laura et Mario Aliphat, 2002. «Organización política de los Itzaes desde el posclásico hasta $1702 »$, Historia Mexicana, 60, 4, pp. 713-748.

De Vos, Jan, 1988. La paz de Dios y del rey. La conquista de la selva lacandona, 1525-1821. México : FCE.

FarRISs, M. Nancy, 1984. Maya Society Under Colonial Rule : The Collective Enterprise of Survival. Princeton, New Jersey : Princeton University Press. 
Freidel, David A., 1992. « Children of the First Father's Skull : Terminal Classic Warfare in the Northern Maya Lowlands and the Transformation of the Kingship and Elite Hierarchies », Mesoamerican Elites. An Archaeological Assessment. Diane Z. Chase y Arlen F. Chase (eds), pp. 99-134. Norman : University of Oklahoma Press.

GARZA, Mercedes de la. (éd.), 1983. Relaciones histórico geográficas de la Gobernación de Yucatán. 2 tomes. México : UNAM.

HAssig, Ross, 2000. « La guerra maya vista a través del altiplano posclásico ». La guerra entre los antiguos mayas. Silvia Trejo (éd.), pp. 159-173. México : INAH.

Jones, Grant D.

1989. Maya Resistance to Spanish Rule: Time and History on a Colonial Frontier. Albuquerque: University of New Mexico Press.

1990. « Prophets and Idol Speculators. Forces of History in the Lowland Maya Rebellion of 1638 » in Vision and Revision in Maya Studies. Flora C. Clancy y Peter D. Harrison (eds.), Abuquerque : University of New Mexico Press, pp. 179-193.

León Pinelo, Antonio de, 1960. Relación que en el Consejo Real de las Indias hizo sobre la conquista de las provincias del Manché y Lacandón el licenciado...., France V. Scholes y E. B. Adams (comps.), Guatemala : Editorial Universitaria.

López De Cogolludo, Diego, 1971. Historia de Yucatán. 2 vols. Graz, Austria : Akademishe Druck.

PiJOAn Carmen et Josefina Mansilla, 1997. « Evidencia de sacrificio humano, modificación ósea y canibalismo en el México prehispánico ». El Cuerpo humano y su tratamiento mortuorio, pp. 193-212. Elsa Malvido, Grégory Pereira et Vera Tiesler (coords.). México : INAH.

Schele, Linda et David Freidel, 1990. A Forest of Kings. New York : William Morrow and Company.

Schele, Linda et Peter Mathews, 1998. The Code of Kings. The Language of Seven Sacred Maya Temples and Tombs. New York : Scribner.

Simmons, Scott E, 1995. « Maya Resistance, Maya Resolve. The Tools of Autonomy from Tipu Belize », Ancient Mesoamerica núm. 6, pp. 135-146.

Tovilla, Martín A., 1960. Relación histórica descriptiva de las provincias de la Verapaz y de la del Manché, escrita por..., año de 1635. Guatemala : Editorial Universitaria.

Villagutierre Y Sotomayor, Juan de, 1933. Historia de la conquista de la provincia del Itzá. Guatemala : Sociedad de Geografía e Historia de Guatemala.

XimÉnez, Francisco, 1973. Historia de la provincia de San Vicente de Chiapa y Guatemala de la Orden de Predicadores, T.V. Guatemala : Sociedad de Geografía e Historia de Guatemala. 
